\title{
Reflections on biological cybernetics: past, present, prospects
}

\author{
J. Leo van Hemmen ${ }^{1}$
}

Published online: 16 April 2018

(c) Springer-Verlag GmbH Germany, part of Springer Nature 2018

In the natural sciences, scientific revolutions do happen, but they hardly ever happen as a kind of discontinuity ${ }^{2}$ in time. Quantum mechanics in the physical world of 1926-28 is still more or less an exception, and an exciting one. Looking back, my time as coeditor-in-chief of Biological Cybernetics from 1999 onwards and as editor-in-chief between 2006 and 2017 was both exciting and smooth, but it nevertheless prompts a few critical thoughts, all the more since the last two decades have seen important new developments in both experimental and theoretical neuroscience and in the ways of presenting them. Following the natural rhythm of time, we will focus on the past, present, and prospects of the journal as such and on what an editor-in-chief can contribute.

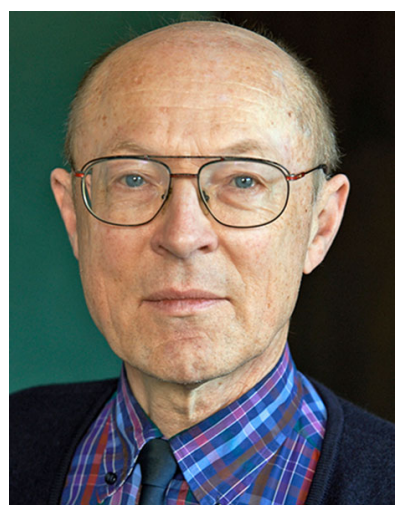

Past What, then, is biological cybernetics, and what makes it so interesting that it deserves its own journal? Biological Cybernetics is the oldest journal in theoretical neuroscience. When it first appeared, there was no need for an explicit definition of the term because Norbert Wiener had carefully defined the notion of cybernetics in 1948 in two epochmaking works (Wiener 1948a,b). In Wiener's own words, cybernetics attempts to find the common elements in the functioning of automatic machines and of the human ner-

$凶$ J. Leo van Hemmen

lvh@tum.de

1 Physik Department T35 \& BCCN - Munich,

Technische Universität München,

85747 Garching bei München, Germany vous system and to develop a theory that will cover the entire field of control and communication in machines and living organisms. Whereas the unification of theories describing the perception, planning, and action of both machines and living organisms is highly desirable from the point of view of developing powerful human-machine interfaces, it is also a highly valuable goal to strive for a deeper understanding of each of them separately and, then, try to unify them. That is, what Wiener formulated 70 years ago remains highly relevant or, to be more fashionable, modern. The goal is a mathematization of neurobiology because without mathematics, no algorithms and, hence, no hardware realization of precisely these mathematical algorithms are possible.

We are on our way, though. What makes me say that? I hope that in these reflections, it is not too presumptuous of me to quote myself a few times as this is within the context of Biological Cybernetics, or BC for short. As I have explained in detail elsewhere (van Hemmen 2007, 2009, 2014), particularly in BC's special issue appearing in 2014 in honor of the great anatomist and neuro-philosopher Valentino Braitenberg, the mathematization of biophysical or, in our case, neurobiological reality is possible only through key concepts. Just as in physics where, as an example, only the key concept of momentum, $\boldsymbol{p}=m \boldsymbol{v}$, with $m$ as mass and $\boldsymbol{v}$ as velocity, gives rise to Newton's second law, $\boldsymbol{F}=\mathrm{d} \boldsymbol{p} / \mathrm{d} t$, which describes mathematically a particle's response to a force $\boldsymbol{F}$, so in neuroscience (Georgopoulos et al. 1986; van Hemmen and Schwartz 2008), the population vector predicts the actual motion direction realized by a group of cortical motor neurons on the basis of perception or intention, and only the learning window (Gerstner et al. 1996; Markram et al. 1997)

\footnotetext{
${ }^{2}$ If the reader thinks that I am starting out by attacking Thomas S. Kuhn's masterpiece The structure of scientific revolutions (University of Chicago Press, Chicago, 1962, 1970, 1996, 2012), I am not. In my opinion, the book makes for wonderful reading but exaggerates scientific reality a bit. Continuity is the rule, discontinuity the exception, and the latter is Kuhn's focus. As an example, through its time-dependent increase and decrease of synaptic efficacies, STDP represented a complete break with existing ideas on learning, but it took at least a decennium before its full importance was appreciated.
} 
can explain spike-timing-dependent plasticity, or STDP for short.

One may call the population-vector code Newton's second law for cortical motor neurons. Of course, there are variations and refinements. Biology and neurobiology are characterized by a universality that is different from that of physics in that a law nearly always holds but has a low (1-2\%) percentage of exceptions "proving" it (van Hemmen 2014); to venture an explanation, by evolution.

Furthermore, one needs to carefully discern, and distinguish, scales (van Hemmen 2014). For instance, and to take two extremes, the natural laws of neuronal dynamics offer us totally different statements from those in psychology. No doubt, there are relations between the two, but their way of understanding and the phenomena they focus on are, so to speak, miles apart, in totally different landscapes. Discovering whether and how laws as generally valid descriptions of behavior exist in their own right and what they look like is what scientific discovery amounts to. The laws that hold on different scales are of course related to but cannot be straightforwardly derived from each other. That is, the assumption that there are different scales with their own laws is the scaling hypothesis. Physics, with mechanics, quantum mechanics, and elementary-particle physics, each with eight orders of magnitude of spatial difference, is merely a prominent example that provides inspiration. And it goes without saying that scales need to be discerned in both space and time; they are in general spatiotemporal.

Those living in Wiener's times did not yet know the key notions needed to obtain mathematical descriptions of perception, neuronal dynamics, and action planning. The idea that different scales, for instance, micrometers for neurons, millimeters for nuclei, centimeters for brain areas, and the outside world for the whole brain plus its external impact, would require a different formalism for each, with different key notions and different mathematics, was unknown to Wiener and other scientists of his day-and long thereafter. The simple lack of appropriate key notions already sufficed to make the mathematics that Wiener imagined to exist a mere illusion. It is this that explains why Wiener's 1948 book, though it introduced biological cybernetics amazingly clearly, did not have the impact it should have had.

The impact of Wiener's imagination came to life half a century later, by which time his fame had waned, mathematical theories had started to be implemented in hardware, and the hardware itself had reached such a state of miniaturization that it allowed for suitable implementation. The key notions, such as neurons as threshold elements, population-vector code, and STDP, which had hitherto been unavailable or had not come to be seen as essential for information processing in 1948, now came into widespread use. Key notions, scaling, and universality (van Hemmen 2014) entered the playing field and changed the theoretical scene completely. On top of that, only by the turn of the century did a first understanding of the notion of action planning and decision-making, which is essential for any motion, appear. The functioning limbs Wiener dreamed of are now being built and do their job with amazing perfection.

By the end of the last century, theoretical neuroscience had finally started to acquire a flexible mathematical apparatus capable of describing neurobiological reality, handling practical problems, and rising to the challenges posed by new problems on the basis of existing tools that had been absent in Wiener's time. And, if I may say so, like Wiener, one of the founding fathers of Biological Cybernetics, I have always seen the journal as one that, on a solid mathematical basis, ought to disseminate the kind of mathematical understanding that is needed to quantify the plethora of fascinating phenomena in neuroscience. Of course, it also behooves the journal to provide a thorough understanding of novel mathematical techniques that are needed to analyze neuronal data, be they of a statistical, dynamical-systems type or of some other, so far unknown, character.

In short, in the 60 years of its flourishing existence as the first journal in computational or, in my opinion more accurately, theoretical neuroscience, Biological Cybernetics has aimed and, I think, entirely succeeded at managing the challenging task of providing a mathematical basis for quantifying and, hence, explaining the fascinating data obtained through experimental neuroscience. It is the intense interaction between experimental and theoretical neuroscience that that has proven most fruitful. Precisely this interaction was, and is, the true foundation of Biological Cybernetics, despite its emphasis on mathematical explanations of neurobiological reality.

Present Pointing out that key notions are essential for quantifying natural phenomena is (nearly always) based on a mechanistic understanding, the key notion in general being closely related to an underlying mechanism. That this is not a tautology is illustrated by Newton's second law, $\boldsymbol{F}=$ $\mathrm{d} \boldsymbol{p} / \mathrm{d} t$. Momentum $\boldsymbol{p}=m \boldsymbol{v}$ has a clear physical intuition to anybody who has driven a car, but that its manner of changing is determined by forces in the simple way indicated by $\boldsymbol{F}=\mathrm{d} \boldsymbol{p} / \mathrm{d} t$ was Newton's great discovery.

Mechanistic means that, founded on the groundwork of our present insights and understanding, one can determine the mechanism behind the way "things" work. For instance, together with Andy Schwartz, I was able to show (van Hemmen and Schwartz 2008) that a population-vector code would predict on the level of perception what an animal is going to do. That is, one takes the math, performs the computations, and explains the available experimental data. Equally important, though, one can now propose new experiments and quantitatively predict the outcomes. The latter idea is still new to biology; most experimentalists still need to get 
used to it and discover its advantages. In nearly all cases, the outcomes continue to surprise but are highly rewarding.

In a mechanistic context, the use of probability is indispensable. The underlying reason for this is simple: We do not know all the factors surrounding an event or a lab experiment that could alter outcomes without our knowing why or without being able to quantify the causes. Because of this lack of knowledge, we must resort to probability theory (Lamperti 1966) so as to mimic the effect of unknown agents that are supposed to modify the behavior of an object of interest. To give it a more positive formulation, probability theory takes into account our lack of knowledge.

In recent times, the Reverend Thomas Bayes (1702-61) has become popular once again. [For background information on him and his work, the interested reader may refer to a two-page introduction by Dale (1989) and the book reviews of Faris (2006) and Zabell (2005).] The key idea of Bayes is conditional probability, a very old notion that in the context of modern probability theory goes back to at least Kolmogorov (1933). In modern mathematical terms, the conditional probability $P(B \mid A)$ of obtaining event $B$, given that $A$ with $P(A)>0$ has occurred, is defined by $P(B \mid A)=P(A B) / P(A)$, where $A B$ is the intersection of the sets, i.e., events, $A$ and $B$. This is quite a natural definition because, once we know event $A$ has happened, the rest must be simultaneous to and, hence, contained in $A$, and we are left with the intersection of $B$ and $A$, viz., $B A$. If $B=A$, then the probability that $A$ will happen given that $A$ happens is bound to be $P(A \mid A)=1$, so that normalization by $1 / P(A)$ is just right. Here is one more argument. If $A$ and $B$ are independent so that $P(A B)=P(A) P(B)$, then $P(B \mid A)=P(A B) / P(A)=P(B)$, a natural result since $A$ does not matter for $B$ 's occurrence.

Under Bayesian analysis, three things need to be constantly borne in mind. First, the question as to whether the brain implements probability distributions remains an open one. After all, sampling distributions is in many senses a costly affair. Second, one uses probability theory from the start, not as a source of background noise. In so doing, one stays away from mechanistic explanations. For an assembly line in a factory, Bayesian techniques are fine, but in nature, where unexpected situations are the rule, the appropriate context of repetition is usually missing. Third, most of the time, such a probabilistic description will make use of various applicable parameters. Choosing the "right" values remains more art than science, so one does well to remember a saying dating back to von Neumann, as quoted by Fermi in a discussion he had with Dyson in 1953, retold by Dyson (2004) half a century later. Fermi asked him, "How many arbitrary parameters did you use for your calculations?" Dyson thought for a moment about the cut-off procedures and said, "Four." Fermi then replied, "I remember my friend Johnny von Neumann used to say, with four parameters I can fit an elephant, and with five I can make him wiggle his trunk." And there the conversation ended.

Open access and Open Choice. In an editorial that appeared a few years ago (van Hemmen 2015), I carefully analyzed the phenomenon usually referred to as open access. It has meanwhile become a huge problem, making the discussion back then more than timely. As scientists, we all need to be very worried. First, scientific publishing was and should be of high quality, with carefully performed peer review, attentive editing, and durable electronic archiving. (No doubt, paper lasts longest.) None of this can be done for free, so someone must pay for it: a library, a reader, the author, or all three. We are now at a point where the burden of coming up with funding for published works is being shifted from libraries to authors. This means that the money traditionally provided through libraries is now given to authors, who must pay for the publication of their work. Instead of a reader buying a book or a library paying for access, interested readers can read some work for free, but then research as a whole suffers budget cuts.

Not all scientists can afford to publish through open access, which prompted Springer to come up with what it calls Open Choice. The author decides whether s/he can spend the money on open access or needs to publish under the aegis of a library because the open-access funds are not there (yet). I think this is the correct way to go about it because it gives authors the freedom to decide how to publish their work. After all, it is the author and not the reader who created the text. This also means that there is no longer a need for mere open-access journals because those who can afford it can publish in the open-access format, and those who cannot are still able to publish; in both cases, the only proviso concerns quality. This is the policy that Biological Cybernetics upholds.

The key problem is now that science is being flooded, to understate the situation, with open-access journals. Every day I receive at least one and usually two "offers" to publish my manuscript in some new journal. Of course, as an active scientist, I have accumulated piles of them and I have even more money to pay for their publication. The deadline is, at the latest, the end of next week, and I'm guaranteed to see my work published the week thereafter, which is a clear sign of a high-quality review process. Of course, this way you can stick to the traditional route: you pay for an advertisement and so have the right to publish. But do you?

Prices vary greatly and are coming down gradually, but the raw number of new open-access journals is overwhelming. Who has the time or capability to verify the virtually uncountable number of publications? No one, of course. All scientists ought to-actually must-read cutting-edge scientific material, but a swelling flood of journals makes it impossible to stay on top of all the new publishing venues. Except for the highest-quality publications, which are read- 
ily identified, scientific dilution, practically homeopathic, is the result. It seriously hampers information transfer and is the problem facing present-day science: the overwhelming flood of new journals with no quality control. Some sort of action must be taken - and soon. This conclusion has become all the more urgent because, even if the flood of manuscripts were to be reviewed, one needs reviewers, and, based on the flood of manuscripts coming across one's desk, conscientious reviewers will be hard or impossible to find (Arns 2014).

Whereas most traditional journals edited by scientific societies and publishers such as Springer have maintained high quality standards, the overwhelming majority of open-access publishers didn't start out with those standards and have yet to impose them. Now, maybe they don't even need to because their clients pay for their ads and so have the right to see their text published. I know, quality checks guarantee, with decent probability, that published works will not contain wrong information. But such verification is, to be blunt, a joke, and here's why: To say that $1+1=3$ is, of course, wrong, but to say that $1+1$ is around 3 is not, strictly speaking, incorrect, so it is (I guess) legitimate to publish. The only conclusion any respectable scientist could come to is this: Journals that aim to provide scientific information must impose stringent quality standards.

Prospects Why do we discuss problems of scientific publishing and ways of solving them? There are no problems, only opportunities. Everybody publishes in arXiv or equivalents what she likes best, and the scientific community can read it for free and append comments, if desired. End of discussion, right?

Where, then, are the publications that are relevant for us? Huge outfits like PLOS ONE may very well publish a decent paper every now and then. It is meanwhile also known that high impact factors are supported by only a few highly cited papers. How, then, can I hope to find what I need in such a gigantic warehouse? Titles don't always provide good clues. There is, however, a far more important drawback. Several people have recently put forth the very argument I just formulated, but these people, I'm afraid, have much more time on their hands than an active researcher working at the cutting edge of science.

When one is actively involved in research, one should be able to trust the papers one reads and be sure that the details are correct. Peer review, as it has been practiced during my time as editor-in-chief of Biological Cybernetics, involves the careful analysis of a manuscript that will uncover sloppy formulations and plain or subtle errors and provide helpful hints regarding style to less experienced authors. Not only do we finally get papers that even reviewers are pleased with but, from a more elevated point of view and far more importantly, through careful peer review and editing, we obtain publications that we as scientists can trust and can pass on to our graduate students without first needing to wade through an ocean of fuzzy comments and discussions as to what is wrong and what's right. In passing, since long, the half-life of BC's papers has exceeded more than 10 years. I frankly acknowledge that the review process is performed by humans who are not always impartial, but the percentage of flawed decisions is small (my personal guess puts it at less than 3 percent). In plain English, active scientists have no time for trivial details and quirky arguments; they require clean, clear, and reliable information on new results obtained by their colleagues.

The transfer of high-quality, novel science will guarantee the future of any critically minded, carefully peer-reviewed, and well-edited journal such as Biological Cybernetics. High quality is a qualifier that does not come cheap, but it is the only one that allows for a smooth functioning of scientific data transfer. In BC's context, mathematics is the very basis of all algorithms and the hardware implementing them, and these together lead to understanding and implementing neuronal functioning that was impeded or absent previously. Since its very beginning, Biological Cybernetics has aimed at publishing papers that lead to an increased mathematical and, hence, quantitative understanding of neuronal activity on all levels, from the microscopically neuronal to the macroscopic. I wish the new editor-in-chief, Benjamin Lindner, much inspiration, wisdom, and success as he guides the journal through the exciting developments in computational/theoretical neuroscience that await all of us.

Acknowledgements As former Editor-in-Chief of Biological Cybernetics it is my great pleasure to thank two people: Dr. Sabine Schwarz (Springer-Verlag, Heidelberg), who always had open eyes and ears for the Journal's needs and with whom I have collaborated truly creatively, and Glenn Corey, who over the many years of my tenure as Editor-inChief critically evaluated my prose as a friend and through his input has made it take off. For hard-core scientists, this takeoff is by no means self-evident (van Hemmen 2013, Appendix B).

\section{References}

Arns M (2014) Open access is tiring out peer reviewers. Nature 515:467 For all data mentioned in the present editorial the reader is referred to Wikipedia, which also offers detailed references

Dale AI (1989) Thomas Bayes: a memorial. Math Intell 11(3):18-19 This little, no-nonsense, essay is, in my opinion, one of the best on Bayes as it is exactly right in pointing out that what one calls Bayes theorem is just noting that Bayes was the first to discover and use the notion of conditional probability

Dyson FJ (2004) A meeting with Enrico Fermi: how one intuitive physicist rescued a team from fruitless research. Nature 427:297

Faris WG (2006) Probability theory: the logic of science. Notices Am Math Soc 53(1):33-42 and references quoted therein

Georgopoulos AP, Schwartz AB, Kettner RE (1986) Neuronal population coding of movement direction. Science 233:1416-1419

Gerstner W, Kempter R, van Hemmen JL, Wagner H (1996) A neuronal learning rule for sub-millisecond temporal coding. Nature 383:7678 
Kolmogorov AN (1933) Grundbegriffe der Wahrscheinlichkeitsrechnung. Springer, Berlin; Foundations of the theory of probability. Chelsea, New York (1950), §4

Lamperti J (1966) Probability. Benjamin, New York (2nd edn 1996, Wiley, New York)

Markram H, Lubke J, Frotscher M, Sakmann B (1997) Regulation of synaptic efficacy by coincidence of postsynaptic APs and EPSPs. Science 275:213-215

van Hemmen JL (2007) Biology and mathematics: a fruitful merger of two cultures. Biol Cybern 97:1-3

van Hemmen JL (2009) Editorial to volume 100 of Biological Cybernetics. Biol Cybern 100:1-3

van Hemmen JL (2013) Vector strength after Goldberg, Brown, and von Mises: biological and mathematical perspectives. Biol Cybern 107(4):385-396 van Hemmen JL (2014) Neuroscience from a mathematical perspective: key concepts, scales and scaling hypothesis, universality. Biol Cybern 108(5):701-712

van Hemmen JL (2015) My science, right or wrong! Biol Cybern 109:1-3

van Hemmen JL, Schwartz AB (2008) Population vector code: a geometric universal as actuator. Biol Cybern 98(6):509-518

Wiener N (1948a) Cybernetics, or control and communication in the animal and the machine. Wiley, New York, and Hermann, Paris. See in particular p. 19

Wiener N (1948b) Cybernetics. Sci Am 179(5):14-18

Zabell SL (2005) Bull Am Math Soc 42(4):555-559 